\title{
Discharge Correlates of Hippocampal Complex Spike Neurons in Behaving Rats Passively Displaced on a Mobile Robot
}

\author{
Vladimir V. Gavrilov, Sidney I. Wiener,* \\ and Alain Berthoz \\ CNRS-Collège de France Laboratoire de Physiologie de \\ la Perception et de l'Action UMR-C 9950, 75231 Paris \\ Cedex 05, France
}

\begin{abstract}
This study investigated location-, movement-, and directional-selectivity of action potential discharges of hippocampal neurons in awake rats subjected to passive displacements in order to estimate vestibular contributions to this activity. Water-deprived rats were habituated to being restrained in a sling mounted on a moving robot. The extracellular activity of single complex-spike cells in area CA1 of the hippocampus was recorded with glass micropipettes in the rats during passive translations, rotations, and immobility. The robot made a standardized series of trajectories starting from each of four corners of a square enclosure surrounded by black curtains. A drop of water was delivered to the rat each time the robot arrived at one designated corner of the arena. The activities of 29 neurons were investigated in 45 recording sessions (16 of which were in total darkness) in four rats. Hippocampal neurons recorded in 31 sessions (9 sessions in the dark) had significant locationselective increases or decreases in firing rate as the rat was passively displaced or immobile within the experimental arena. In 20 sessions (6 in the dark) direction-selective discharges were found when the rat was in the corners. In six sessions, cells discharged selectively during movement initiation or termination. These data suggest that information essential for path integration is present in the hippocampus and that inertial cues could play a vital role in hippocampal spatial functions. These results resemble those of O 'Mara et al. ([1994]] Neurosci 14:6511) using the same protocol in macaques, suggesting similarities in hippocampal processing and function. H ippocampus 8:475-490, 1998. ๑1998 Wiley-Liss, Inc.
\end{abstract}

KEY WO RDS: vestibular; passive displacement; recordings; robot

\section{INTRODUCTION}

Location-selective discharges have been shown in hippocampal neurons of behaving rats (reviews: 0 'K eefe, 1979; M uller, 1996; W iener, 1996) and monkeys (O no et al., 1993), but the physiological mechanisms that

Grant sponsor: EC ESPRIT MU CO M ; Grant numbers: 3149 and 6615; Grant sponsors: Human Frontiers, EG Human Capital and Mobility Program, CN RS Programme Cognisciences, and Fondation pour la Recherche Médicale.

Vladimir Gavrilov's current address is: Institute of Psychology, Russian Academy of Sciences, 129366 M oscow, Russia

*Correspondence to: Sidney I. Wiener, CN RS-Collège de France LPPA, 11 pl. Marcelin Berthelot, 75231 Paris Cedex 05, France.

E-mail: sw@ccr.jussieu.fr

Accepted for publication 1 M ay 1998. generate them have yet to be determined. 0 'K eefe and Conway (1978) showed that multimodal environmental landmark cues can serve as primary referents for determining those locations (called firing fields) where the firing rates of these cells are elevated. Hippocampal locational representations can also be updated by cues concerning self-movements (e.g., optic field flow, inertial/ vestibular and motor feedback signals). This could serve as a neural basis for path integration, the ability of animals that have followed a circuitous route to return directly to the original point of departure (discussed in Wiener and Berthoz, 1993).

Recordings from unrestrained rats in a rotating arena show that hippocampal firing fields can be updated to remain stationary with respect to the gravitoinertial Earth-based reference frame (W iener et al., 1995; Sharp et al., 1995). The cue modalities involved in this updating process could include vestibular, proprioceptive, and motor command signals. In the latter experiments the rats were freely standing. Thus the passive rotations could have evoked postural reflexes or locomotor reactions, possible cues for the angle of rotation. Unfortunately, this was not controlled for in these experiments since the animals were rotated in darkness. To test for evidence for inertial cue influences on location-selective hippocampal responses in the absence of information generated by voluntary movements, we recorded extracellular activity in restrained awake rats as they were passively displaced on a mobile robot. Since similar experiments performed on restrained awake macaques have shown location- and movement-selective discharges (O no et al., 1993; O 'M ara et al., 1994) another goal here was to determine whether rat hippocampal neurons would show comparable responses.

The methodology of the present study bears a superficial resemblance to the work of Foster et al. (1989). They recorded firing fields of hippocampal neurons of freely moving rats, then securely restrained the animals in a towel, and tested the same neurons as the rat was carried through the firing fields. Under these conditions the 
neurons maintained only "a very slight residual specificity." However, if the rat was only lightly restrained and the same procedure was repeated, the location-selective discharges were maintained at their original strength. The interpretation of these results was that location-selective discharges occur only if the rat is prepared to move, and presumably navigate, in the environment.

In contrast to the Foster et al. (1989) experiments that tested changes in firing field properties in mobile and restrained animals in the light, the present experiments aimed to test, in restrained rats only, for evidence for influences of inertial cues on locationselective discharges. It was not possible to maintain stable recordings of the same cell in restrained and unrestrained conditions with the driver used here. In the present study, the awake rats were trained to receive a water reward when transported by the robot to a fixed location in the arena. This was intended to motivate the animal to attend to its position in the environment despiteits immobility. T hen we applied a comprehensive series of analyses for evidence of inertial cue influences on location, directional, and movement correlates in the hippocampal neurons of the restrained animals.

\section{MATERIALS AND METHODS}

Experiments were performed on four adult male Long-Evans hooded rats weighing about $350 \mathrm{~g}$. Some of these results have been published in abstract form (Gavrilov et al., 1994). The rats werehoused in individual cages in an animal care facility on $\mathrm{a} 2 \mathrm{~h}$ light/dark cycle and received food ad libitum. Experiments were performed within a square arena ( $3 \mathrm{~m} \times 3 \mathrm{~m}$ ) with black curtains along the sides and suspended from above (Fig. 1). The arena corners will be referred to as numbers 1, 2, 3, and 4 and the walls as west, north, east, and south. The principal salient cue in the arena was an illuminated translucent white ball $30 \mathrm{~cm}$ in diameter.
This was suspended from the ceiling just above the eye level of the rat in corner 2 (where water rewards were given).

\section{Training}

A water deprivation schedule was started 2 days prior to the beginning of training. Body weight was maintained at at least $80 \%$ of normal weight. Each rat was gradually trained over the course of a 1 month period to be restrained, suspended, and transported in a cloth sling mounted on a custom-built apparatus resembling a small stereotaxic instrument. This arrangement permitted all four limbs and the head to protrude freely. A normal alert posture was assumed. Training consisted of three stages: initial adaptation to restriction in the sling, then adaptation to passive displacements while restrained on the robot, and finally, after surgical implantation of the electrodes, displacements with the head fixed. At first the rat was fixed in the sling on the motionless robot for at least 30 min each day and a drop of water (about $50 \mu \mathrm{l}$ ) was delivered automatically every $10 \mathrm{~s}$. The water was delivered in a small reservoir mounted below the muzzle of the rat. This period of adaptation lasted about 5- 7 days. When the rat had learned to repose quietly under restraint and to drink water from the cup, the next stage of training was started. In general, lapping the water was considered a behavioral criterion showing that the rat was ready for the following stage of training. At this stage the rat was passively displaced on the robot, and a drop of water was delivered only after arrival in the corner of the arena containing the illuminated white ball. U sually it took a few days for the rat to adapt to being moved on the robot, and we continued training daily (one or two sessions) for at least another week. D uring training, the set of trajectories and identity of the rewarded corner were the same as those used during recordings. $\mathrm{O} n$ the third day after recovery from surgery we repeated training sessions for at least 2- 4 days with the head fixed. Then recordings were performed on weekdays, permitting the rats to rehydrate at the beginning of each weekend. Although the trained rats could be
FIGURE 1. Schematic of the experimental apparatus and protocol. Above, The experiments were conducted in a $3 \mathrm{~m} \times 3 \mathrm{~m}$ square area surrounded by black curtains at the sides and top. The rat was restrained in a sling on top of the robot. The robot moved along the trajectories indicated by the lines with double arrowheads. The principal visual cue was a white ball suspended in one of the corners. The arrival of the robot at this point was detected by a vertically oriented photodetector. Red and blue lamps mounted on an aluminum frame on the robot (not shown) were detected by a video camera located at the center of the roof of the enclosure. Actual trajectories were made in a 0.5 (or 1.5 ) $\mathrm{m}$ sided square near the corner containing the visual cue. Below, The trajectories are shown in overhead views. The corners of the enclosure are indicated by numbers 1-4; corner 2 is circled, indicating that this was the location of the illuminated sphere cue in all experiments. The compass directions are indicative of the absolute coordinate frame, and in all figures "north" is toward the top of the page. A: The locations occupied by the robot and the rat along the course of a recording session. In these data from an actual recording session of a single CA1 complex spike cell, an X indicates the position then occupied by the rat each time the cell discharged. The size of each $X$ is proportional to the number of spikes in a $\mathbf{5 0} \mathrm{ms}$ interval. This cell fired at the same rate thoughout the arena and was not location-selective since the $X s$ are found at the same density in all positions. (The path width deviated slightly along certain walls of the arena). B: A series of trajectories originated in each of the four corners. The robot advanced with the rat facing forward (F). After a $180^{\circ}$ turn clockwise, it returned to the original corner (R) and again to the second corner (F). The robot then carried the rat facing the direction opposite that of the previous translations back to the original corner (B). The forward trajectories were also repeated at double velocity. $\mathrm{C}$ : Then the robot made a triangular trajectory and proceeded to the next corner to start again. At the end of each translation or rotation, the robot paused for two $S$. In D, E, and $F$, the directions of each of the three movement types is shown. $D$ : Linear trajectories were along walls and diagonals in both directions. E: In each of the corners the robot rotated clockwise and counterclockwise. F: At intervals throughout the recording session, the robot paused and remained immobile in each of the corners while directing the head of the rat in each of six possible orientations. 

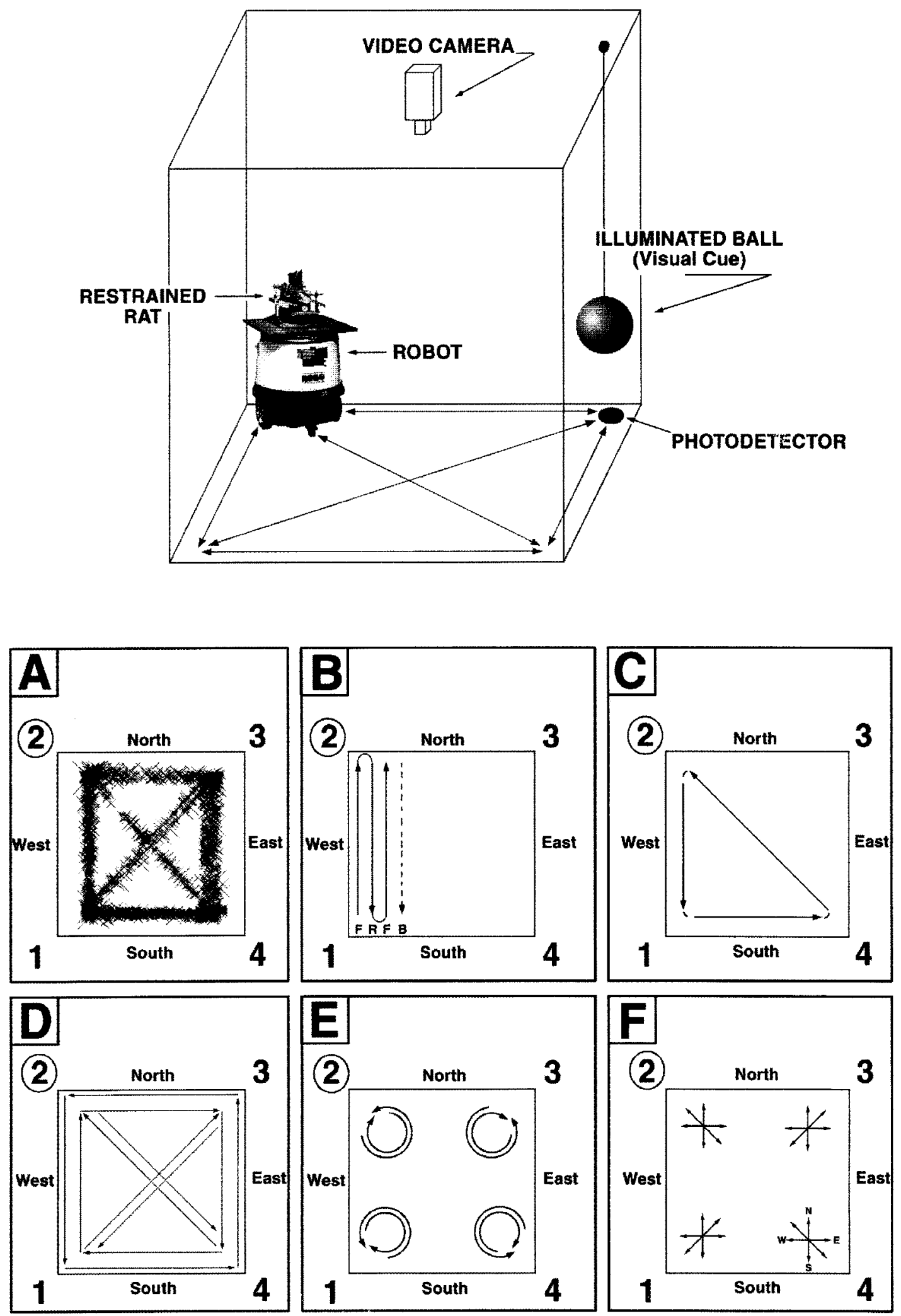

Figure 1 
maintained in the sling for several hours, the head fixation was limited to the shortest periods possible.

\section{Apparatus}

The rats were passively translated and rotated on a mobile computer-driven robot (in the form of a $45 \mathrm{~cm}$ diameter, $50 \mathrm{~cm}$ high cylinder; Robosoft, Bayonne, France). The rats were re strained in a sling held on a custom-made apparatus resembling a stereotaxic instrument. This was contained within a box-like aluminum frame (dimensions: $34 \mathrm{~cm}$ high, $44 \mathrm{~cm}$ long, and 41 $\mathrm{cm}$ wide) attached to the top of the robot. Since the frame was larger than the hammock assembly, during dark sessions a black cloth could be placed over the rat in the hammock while leaving the outer aluminum frame exposed.

\section{Motion Parameters}

The robot was programmed to accelerate and decelerate in $1 \mathrm{~s}$; movements were at constant velocities. The actual sequence of the robot's displacements within the arena are represented schematically in Figure $1 B$ and $C$. The robot first made a series of trajectories between corners 1 and 2 and then repeated the same movements between other pairs of corners in sequence. In this way the rat was displaced in the same manner in each quadrant of the arena. In general the sequence consisted of alternating translations ( $\sim 5-15 \mathrm{~s})$ and rotations ( $\sim 2-3 \mathrm{~s})$ separated by pauses of about $2 \mathrm{~s}$. Pauses occurred only in the corners. A typical recording session consisted of a standard series of 216 movements and pauses in order to facilitate analytical comparisons. In the first two rats, the robot moved within a $0.5 \mathrm{~m} \times 0.5 \mathrm{~m}$ square area, and the translations were at 50 or $100 \mathrm{~cm} / \mathrm{s}$. In the second two rats, the square area for displacements had $1.5 \mathrm{~m}$ sides, and translations were at $100 \mathrm{~cm} / \mathrm{s}$ only. Trajectories were immediately adjacent to the north and west walls of the arena but not the south and east walls. For ease of presentation, all the trajectories are indicated in the corresponding figures and text as also following the south and east walls. At the corners, the robot made rotations clockwise (CW) or counterclockwise (CCW) of magnitude $90^{\circ}$, $135^{\circ}$, or $180^{\circ}$ with an angular velocity of $50 \mathrm{deg} / \mathrm{s}$. Each experimental session lasted about $17 \mathrm{~min}$.

The movement sequences were repeated in light and in darkness to test for location- and orientation-selective discharges in the dark, an evidence for integration of self-movement signals to update spatially selective firing of hippocampal neurons. In "light conditions," a curtain on the north wall of the arena was opened permitting a large number of objects in the illuminated room outside the arena to be seen. For experiments performed in darkness, all lights were turned off, all (black) curtains of the arena were closed, and the sling and headholder apparatus holding the rat was covered with a piece of darkroom curtain. This covering effectively blocked all visual cues since it draped down to the base of the platform that held the apparatus on the robot. W hile the rat was being installed in the apparatus for the darkness session, sufficient cues were available for it to be oriented with respect to the arena. The presentations of the water rewards at corner 2 may have served as a positional cue for the rat. The neurons that were recorded in more than one session have the same numerical identifier, but differ by the letters a, b, or c prior to the last digit. Cells with numbers identical except for the last digit were discriminated from the same electrode (detailed below).

\section{Surgical Procedure}

Prior to surgery, rats were tranquillized with $0.1 \mathrm{ml}$ xylazine (Rompun, 2\%) followed by an intraperitoneal injection of 60 $\mathrm{mg} / \mathrm{kg}$ pentobarbital for anesthesia. The skull was exposed, and five tiny screws were anchored in it to secure two large screws adapted to maintain a light-weight lucite platform (Korshunov, 1995), which would be installed later, just prior to each recording session. A hole of diameter $1.4 \mathrm{~mm}$ was made in the skull (AP - $3.8 \mathrm{~mm}, \mathrm{ML} 1.5 \mathrm{~mm}$ relative to bregma; Paxinos and Watson, 1986). Then the exposed brain surface was cleaned daily and covered with sterile vaseline. A screw in the occipital bone served as an electrical ground. A Teflon insulated stainless steel wire (100 $\mu \mathrm{m}$ diameter) was used as a reference electrode, and this was permanently implanted at a depth of $2.0 \mathrm{~mm}$ at AP -4.8 $\mathrm{mm}, \mathrm{ML} 2.4 \mathrm{~mm}$ relative to bregma and was oriented at a $10^{\circ}$ angle rostromedially (a distance of $1-1.5 \mathrm{~mm}$ from the recording site).

\section{Electrodes}

Glass micropipettes filled with a $2.5 \mathrm{M}$ solution of $\mathrm{NaCl}$ (impedance $0.5-10 \mathrm{M} \Omega$ at $1 \mathrm{kHz}$ ) were used for simultaneous recordings of impulse activity of complex-spike neurons and local electroencephalography (EEG) (for the latter results see $G$ avrilov et al., 1995, 1996). The microelectrode was installed acutely just prior to each recording session with a removable microdrive (Korshunov, 1995) adapted here for glass micropipettes.

\section{Experimental Protocol}

Prior to each recording session, the brain surface was cleaned. Then the lucite platform was attached to the large screws on the headstage. The lucite platform was a support for the light-weight electrode driver (Korshunov, 1995), as well as for electrical connectors. It also had receptacles for bars (configured somewhat like stereotaxic earbars) that in turn were fixed to the same apparatus that supported the sling that held the body of the rat. 0 utside the experimental arena the awake rat was then placed in the sling and headholder. The electrode driver was then installed. The electrode was advanced in 10-40 $\mu \mathrm{m}$ steps by rotating the head of the microdrive. All recordings were made at sites that had a well-isolated neuron showing typical complex-spike activity (with a decrement of the amplitude of spikes in the burst and approximately 5 ms interspike intervals) (Fox and Ranck, 1981; Christian and D eadwyler, 1986). W hen a complex spike cell was found, the sling and headholder assembly holding the rat was attached to the robot. To ensure stability, recordings were started after a 20 min waiting period.

Since the electrodes were mounted eccentrically in the cylindrical driver, the entry site into the brain could be changed for each penetration by rotating the driver prior to replacing it on the 
headstage. All penetrations were within a $0.4 \mathrm{~mm}$ radius of the center of the hole in the skull, and all complex spike recordings were made at a depth of approximately $2 \mathrm{~mm}$ from the brain surface.

\section{Data Acquisition}

Brain electrical activity was passed first through field-effect transistors (FETs) configured for zero gain, and then a differential AC amplifier (model 1700, filter settings 300-5,000 Hz; A-M Systems, Everett, WA) and recorded on an analog FM tape recorder along with the behavioral data. In post hoc analyses, the recordings were entered on a 386SX personal computer with a unit waveform discriminator and an interface (model 1401; Cambridge Electronic D esign, Cambridge, UK). The template matching device (SPS 8701, Prospect, Australia) was operated in the "fixed" template mode (as opposed to "adaptive" templates that accept changes in the waveform along the course of the recording session). In all transfers of the recorded signals from the tape recorder to the computer, the template matcher permitted the waveforms to be continuously monitored to verify accepted and rejected impulses. The criteria for identification of complex spike cells included duration of the unfiltered unit waveforms exceeding $0.5 \mathrm{~ms}$ and average firing rates less than $10 \mathrm{impulses} / \mathrm{s}$ (Fox and Ranck, 1981). In virtually all cases we observed spike bursts with interspike intervals of about $5 \mathrm{~ms}$ (e.g., Figs. 3C, 4C, 5D).

\section{Data Analysis}

A video system (described in Wiener, 1993) monitored the displacements of the robot by tracking the positions of a red and a blue light bulb mounted at the upper part of the box-like frame attached to the top of the robot (described above in the Apparatus section). The red and blue signals were tracked to determine the exact timing of the movements of the robot for synchronization of the unit discharge data since the robot software gave inaccurate timing and odometry signals.

Unit discharges were synchronized with the onsets of translations, rotations, and pauses in the respective corners of the arena. For each neuron, analyses tested for changes in discharge rate during translations along the respective walls (column 3 of Table $1)$. D ata recorded during rotations or pauses at the respective corners were also compared for location-selectivity (columns 4 and 7 of Table 1). M odulation of discharge rate according to the heading direction of the rat during pauses in the corners (facing east, for example) was tested along with location-selectivity during pauses in a two-way ANOVA (columns 8 and 9 of Table 1). $O$ verall location-selectivity was tested by comparing the average firing rates in data collected while the robot was in the respective corners regardless of the ongoing movement (column 6 of Table 1- Place). Selectivity for approaches to the respective corners was tested in data recorded during translations along the walls and the diagonals (column 5 of Table 1). A final analysis examined changes in discharge rate at the beginning and end of translations by combining data recorded along all four arena walls. It compared firing rates between 2 s periods at four phases of the translations along the walls: immediately prior, at the beginning, and prior to the end, as well as the pause after the movement (column 10 of Table 1). The movements with the head facing toward or away from the direction of translation were analyzed separately to distinguish directional vestibular influences.

Separate one-way analyses of variance (AN O VAs) were required for each of the above analyses (except pauses) since they involved different data samples. For significant outcomes (the criteria for significance in this study was $P<0.05)$, Student-N ewman-Keuls post hoc analyses were applied. For eight incomplete sessions not reported here, t-test analyses were performed on the available data, and these were consistent with the present results. The CED (C ambridge, UK) Spike2 macro environment was used for some data analyses. Data were also exported for statistical analysis programs written in PASCAL by S. Schmied.

\section{Histological Procedure}

To mark the vicinity of recording sites, the rats were heavily sedated with pentobarbital and a $20 \mu \mathrm{A}$ D C current was passed through a tungsten recording electrode for $5 \mathrm{~s}$. After a waiting period to permit glial reaction, the rats were euthanized with an overdose of sodium pentobarbital and perfused with $0.9 \% \mathrm{~N} \mathrm{aCl}$ followed by $4 \%$ formalin solution. Brains were conserved in $30 \%$ sucrose solution, then frozen, cut in $50 \mu \mathrm{m}$ sections, and stained with cresyl violet for reconstruction of the recording zone.

\section{RESULTS}

The activities of 29 neurons were investigated in 45 recording sessions (16 of which were carried out in total darkness) in four rats (Tables 1, 2). Thirteen of the neurons could be recorded in only a single session, 13 were recorded in light and in dark conditions, and 2 other neurons were also recorded for a third session in the light. H ippocampal neurons recorded in 31 sessions ( 9 of these were in the dark) had location-selective discharges: there were significant increases or decreases in firing rate as the rat was passively displaced (translations or rotations) or immobile in certain parts of the experimental arena. These data are presented in detail in Table 1 and are summarized in Table 2. In 20 of the sessions ( 6 in the dark) direction-selective discharges were found in one or more corners. Neuronal discharges selective for the beginning or end of movements were found in six sessions. The majority of the cells had more than one correlate from these categories; thus the latter numbers do not sum up.

\section{Spatially Selective Discharges During Translations}

In translations along the sides of the experimental area, significant increases or decreases in firing rate were found in 10/29 sessions recorded in light and in 5/16 sessions recorded in 
TABLE 1.

Results From ANOVAs of Directional and Positional Selectivity in Firing Rate of Single Hippocampal Neurons +

\begin{tabular}{|c|c|c|c|c|c|c|c|c|c|}
\hline Session & $\begin{array}{c}\text { Light/ } \\
\text { dark }\end{array}$ & Translation & $\begin{array}{l}\text { Corner } \\
\text { rotation }\end{array}$ & $\begin{array}{c}\text { Corner } \\
\text { approach }\end{array}$ & Place & $\begin{array}{l}\text { Corner } \\
\text { (pause) }\end{array}$ & $\begin{array}{c}\text { Direction } \\
\text { (pause) }\end{array}$ & $\begin{array}{l}\text { Direction } \\
\text { X corner }\end{array}$ & $\begin{array}{l}\text { Start, end } \\
\text { movement }\end{array}$ \\
\hline 10127 & 1 & & & & * & * & $*$ & $* *$ & \\
\hline 12108 & 1 & & & & & & & & \\
\hline $1210 c 8$ & 1 & & & & & & & & \\
\hline $1412 b 8$ & $\mathrm{~d}$ & $* *$ & & & $* *$ & $* *$ & $* *$ & $* *$ & \\
\hline 15129 & 1 & $* *$ & & $* *$ & $* *$ & & & & \\
\hline 16077 & $\mathrm{~d}$ & & & & & & & & $* *$ \\
\hline 16125 & 1 & & & & $* *$ & $* *$ & $* *$ & & \\
\hline 17127 & 1 & $*$ & & & $* *$ & & $*$ & $* *$ & \\
\hline 18109 & 1 & $* *$ & & $* *$ & $* *$ & & & & \\
\hline 1810b9 & $\mathrm{d}$ & $* *$ & & $* *$ & $* *$ & & & & \\
\hline 20108 & 1 & $* *$ & $* *$ & & $* *$ & * & $*$ & $*$ & \\
\hline $2010 b 8$ & $\mathrm{~d}$ & & & & $* *$ & * & * & $*$ & \\
\hline $2010 c 8$ & 1 & & & & & $*$ & $*$ & $* *$ & \\
\hline 2059 & 1 & $* *$ & & $* *$ & $* *$ & $* *$ & & & \\
\hline $205 a 9$ & $\mathrm{~d}$ & & & & & & & & \\
\hline 22129 & 1 & & & & $*$ & & & & \\
\hline $2307 f 7$ & 1 & $* *$ & & & & $* *$ & * & $*$ & \\
\hline $2307 s 9$ & $\mathrm{~d}$ & & & & & $*$ & * & $* *$ & $* *$ \\
\hline $2412 \mathrm{a} 5$ & 1 & $* *$ & & & & & & & \\
\hline 27109 & 1 & & $* *$ & & $* *$ & & & & $* *$ \\
\hline 2710b9 & $\mathrm{d}$ & & & & & & & $* *$ & $* *$ \\
\hline 2907f9 & 1 & & & & & & & & \\
\hline 30117 & 1 & & & & & $*$ & * & $*$ & \\
\hline 30118 & 1 & $* *$ & & & & & & $* *$ & \\
\hline 3011b7 & $\mathrm{d}$ & $*$ & $* *$ & & $* *$ & $* *$ & & & \\
\hline 3011b8 & $\mathrm{d}$ & & & & & & & & \\
\hline 3127 & 1 & $*$ & & & & & & & \\
\hline $505 a 9$ & 1 & & & & & & & & \\
\hline 5105 & 1 & $* *$ & & $* *$ & $* *$ & $* *$ & & & \\
\hline $510 b 5$ & $\mathrm{~d}$ & & & & $* *$ & & & & \\
\hline 6055 & 1 & & & & & & & & $* *$ \\
\hline 6057 & 1 & & & & & * & $*$ & $*$ & \\
\hline 6058 & 1 & & $* *$ & & & & & $* *$ & \\
\hline $605 b 5$ & $\mathrm{~d}$ & & & & & & & & \\
\hline 6105 & 1 & & & & & $* *$ & $*$ & $* *$ & \\
\hline $610 b 5$ & $\mathrm{~d}$ & & & & & & * & $*$ & \\
\hline $610 c 5$ & 1 & & & & & * & * & $* *$ & \\
\hline 7105 & 1 & & & & & & & $* *$ & $* *$ \\
\hline $710 b 5$ & $\mathrm{~d}$ & $* *$ & & & & & & & \\
\hline 8107 & 1 & & & & $* *$ & & & & \\
\hline $810 \mathrm{~b} 7$ & $\mathrm{~d}$ & & & & & & & & \\
\hline 8129 & 1 & & & & & $*$ & * & $* *$ & \\
\hline $812 b 9$ & $\mathrm{~d}$ & & & $* *$ & & * & $*$ & * & \\
\hline 9059 & 1 & & & & & & & & \\
\hline $905 b 9$ & $\mathrm{~d}$ & $* *$ & & $*$ & $* *$ & & & & \\
\hline
\end{tabular}

Single asterisks indicate a significant $(P<0.05)$ ANOVA result. Double asterisks indicate that at least one of the post hoc analyses was also significant. Results in the third column compare firing rates during linear translations along the west, north, east, or south walls. In the fourth column are comparisons between respective corners during rotations. The "Corner approach" column compares data recorded as the robot approached the respective corners from any direction. The "Place" column tests for differences in data recorded during rotational and translational displacements as well as during pauses. Columns 7 and 8 test for single factor effects in the ANOVA of corner position or heading direction (data recorded in all four corners while the rat was oriented in the respective directions), and the ninth column is the interaction of the two. The final column shows results of one-way ANOVA of firing rates during recordings during the pause prior to linear translations (1), the initial second of movement (2), the final second of movement (3), and the pause after the end of movement (4). In the latter analyses, data were summed for translations along all four walls of the arena. 
TABLE 2.

Summary of Recording Sessions With Significant Results in the ANOVAs for Spatial, Directional or Movement Correlates in Light or Darkness*

\begin{tabular}{lccr}
\hline ANOVA factor & In light & In darkness & Total \\
\hline Translation along walls & 10 & 5 & 15 \\
Corner rotation & 3 & 1 & 4 \\
Corner approach & 4 & 3 & 7 \\
Place (all movements) & 11 & 6 & 17 \\
Corner (pause) & 12 & 6 & 18 \\
Direction (pause) & 11 & 5 & 16 \\
Corner X direction & 13 & 6 & 19 \\
Start or end of movement & 3 & 3 & 6 \\
Total no. of sessions & 29 & 16 & 45 \\
\hline
\end{tabular}

*Numbers are the sum of cases in Table 1 containing one or two asterisks. The totals at the bottom are not sums of the columns because individual neurons had multiple correlates (see Table 1). The first entry in this table should thus be read " 10 of 29 neurons recorded in the light had significant ANOVA results for comparisons of activity recorded during translations along the respective walls." For comparisons between light and dark recordings of the same neuron, refer to Table 1.

darkness (Table 1, column 3-Translation). In each of these recordings, the results of the post hoc analyses (not shown) indicated that there was a gentle gradient of position selectivity. This contrasts with the small circumscribed regions associated with hippocampal complex spike activity found in unrestrained rats. Three representative examples are shown.

Figure $2 \mathrm{~A}$ shows the spatial distribution of discharges of a complex spike cell. In the session recorded in darkness (right), the one-way ANOVA was significant for comparisons of linear movements along the four respective walls. (This neuron also showed other significant results in the AN OVA of data from the two sessions.) The histograms in Figure 2B show the average firing rates during the linear trajectories in these two sessions. $D$ ata from the same neuron recorded in the light are shown to the left. Although the same tendencies are apparent, the AN OVA results for location-selectivity during translations in the light session were not significant. Raster displays of these data (not shown) revealed that in the dark, the cell discharged on $7 / 10$ trials along the east wall, $1 / 10$ on the west wall, and $2 / 10$ trials along the north wall. In the light the cell discharged on $5 / 10$ trials during trajectories along the east wall, but $0 / 10$ on the west wall and $1 / 10$ on the north wall. The chi-square analysis showed no significant difference between these distributions $\left(\chi^{2}=0.71, d f=3, n . s.\right)$. For this neuron the discharge correlates during linear translations along walls in the light and darkness sessions bear some resemblance.

The neuron of Figure 3 had an exceptionally low firing rate $(<0.1 \mathrm{impulse} / \mathrm{s})$. N evertheless the discharge rate was significantly higher for translations along the north wall than along the south and east walls. These location-selective discharges occurred during both low- (left column in Fig. 3A) and high-velocity movements (right column). This is confirmed in the plot of the spatial distribution of discharges shown in Figure 3B. The activity of this neuron was recorded in darkness only.

In contrast, the neuron of Figure 4 had a spontaneous discharge rate that varied as the rat was displaced along the respective walls. The average firing rate was about 5 impulses/s during linear trajectories along the north, west, and south walls; along the east wall, the rate was only about 2 impulses/s (Fig. 4B). T his tendency was observed for slow (left column of Fig. 4A) and rapid (right column) displacements. This neuron's location-selectivity hence manifested as a change in the rate of tonically sustained firing, in contrast to the phasic increases in firing rate found in the cells of Figures 2 and 3.

The overall activity profile of the neuron of Figure 5B suggests that it had location-selective discharges as the rat was displaced along the west and north walls and also in a region near the center during diagonal trajectories. This cell was recorded only in the light. In Figure $5 \mathrm{~A}$ and $\mathrm{C}$ the data from this same neuron have been regrouped according to approaches to each of the respective corners. The average firing rate of this neuron rose from a background level of about $0.1 \mathrm{impulse} / \mathrm{s}$ up to $0.8 \mathrm{impulse} / \mathrm{s}$ during approaches to corner 2, but the ANOVA for corner approaches for this cell had a P level of only 0.1 . H owever, the results of the two-way AN OVA for pauses in the corners, and the "place" AN OVA, which combined linear movements, rotations, and pauses for the respective corners, were both significant. In these two analyses the post hoc tests showed firing rates recorded in corner 2 to be significantly greater than in each of the other corners. However, this position selectivity covers a wider region than usually found in dorsal hippocampal neurons recorded in freely moving rats. This was a general observation for all the neurons with significant results in this test. Among these neurons there was no preference for any particular part of the arena.

\section{Position and Direction Selectivity During Pauses}

In 17 sessions, neurons showed significant differences in discharge rate as the robot paused in the respective corners (Table 1, column 7-Corner pauses) Like the cells described above, these had gentle gradients of spatial selectivity. While significant direction-selectivity (Table 1, column 8) was found in 16 sessions, this was almost al ways accompanied by a significant $D$ irection $\times$ Corner AN O VA result (Table 1, column 9). Thus the supposed direction-selectivity was actually due to a very strong response at only one or two corners (also confirmed by post hoc tests). This contrasts with the activity of head direction cells of the postsubiculum or dorsal striatum, which maintain the same selectivity as the rat occupies all different parts of the environment (Taube et al., 1990; W iener, 1993).

In the 17 recording sessions of cells with location-selectivity during pauses, the areas associated with selective increases or decreases in firing rate seemed to be equally well distributed in all parts of the arena. In general, for the various location-selectivity analyses, there appeared to be no overall tendency among the 

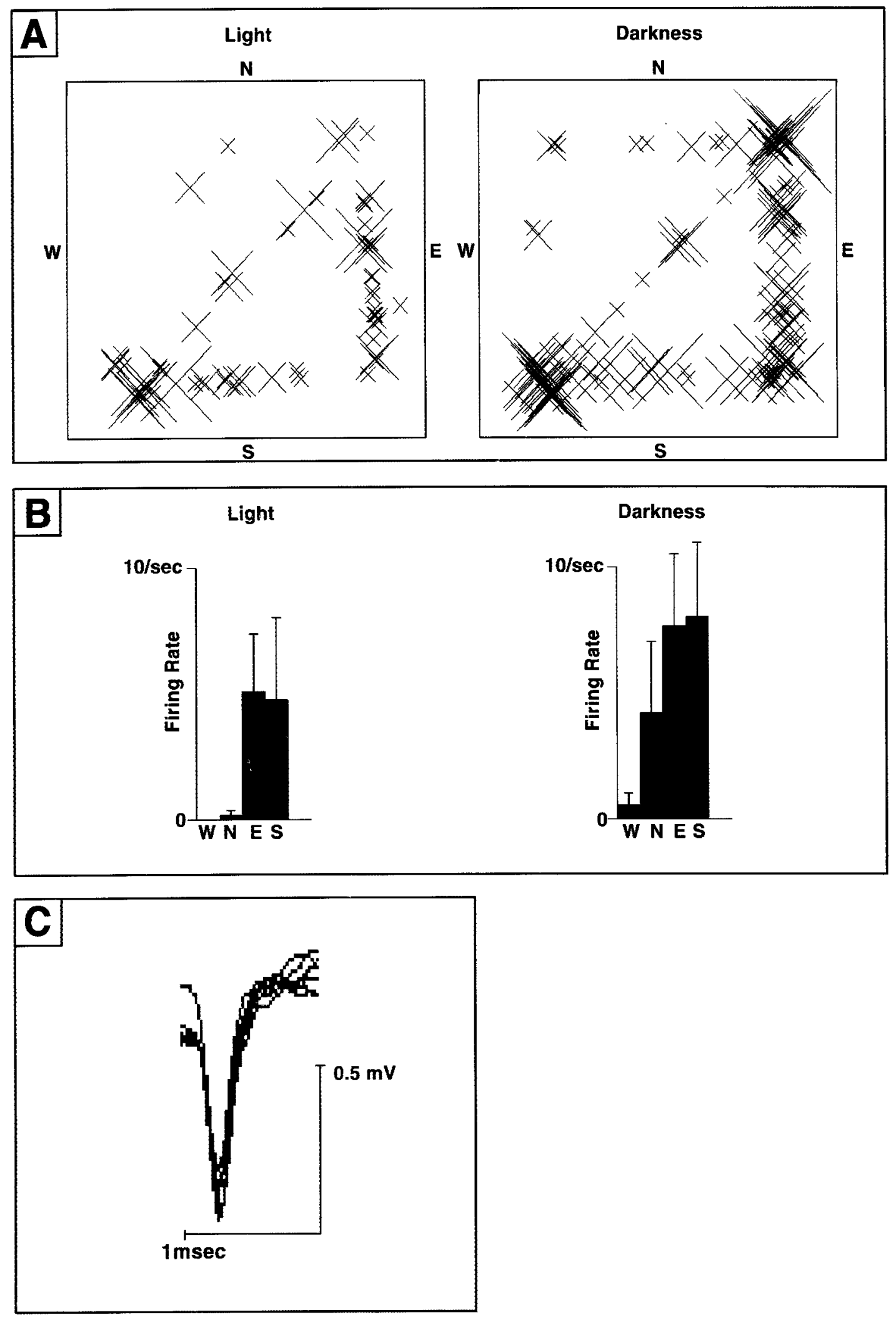

FIGURE 2. Location-selective discharges of a CA1 complex spike cell in a rat passively transported in light and dark conditions. Above, $\mathbf{O}$ verhead schematic views of the distribution of unit discharges in the arena according to the format in Figure 1A. The unit discharged primarily when the rat was transported along the south and the east walls and when it occupied the southwest corner. The "Light" trace at left was recorded with the cues illuminated. The subsequent "D arkness" recording session for this same unit took place with the cue lighting turned off and the animal completely covered with an opaque black cloth. B: The firing of this neuron averaged over all linear trajectories along the walls. Error bars indicate the standard error of the mean. In the dark, discharge rates for trajectories along the east and south walls were both significantly greater than west. C: Several superimposed digitalized waveforms of this unit. (30117 and 3011b7) 

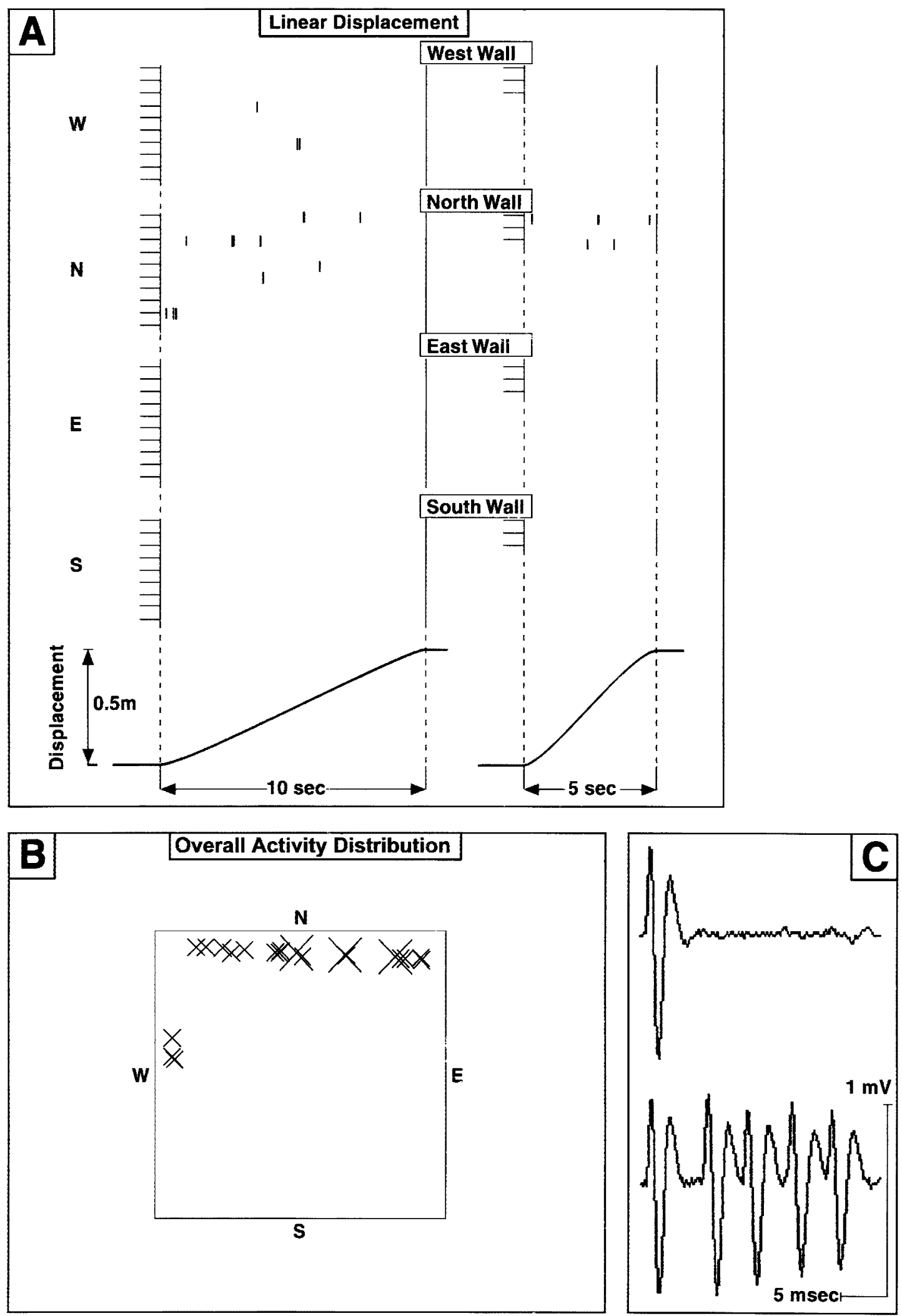

FIGURE 3. Location-selective discharges of a different CA1 complex spike cell recorded in dark conditions. A: Raster displays of unit discharges during translations along the respective walls. Forward and backward movements are included. Slow translations are to the left and faster ones are in the right column. Each row indicated by a horizontal hashmark at the very left represents a single trajectory; vertical rasters indicate the timing of action potentials. The scale below indicates the time course of the displacements along the four walls. This unit discharged rarely, but when it did, this occurred primarily as the rat was translated along the north wall. B: The locations where this unit discharged (same format as Fig. 2C). As shown in $A$, the discharges occurred most often as the rat was transported along the north wall. C: Typical filtered, digitized waveforms from this cell. (cell 1412b8) 

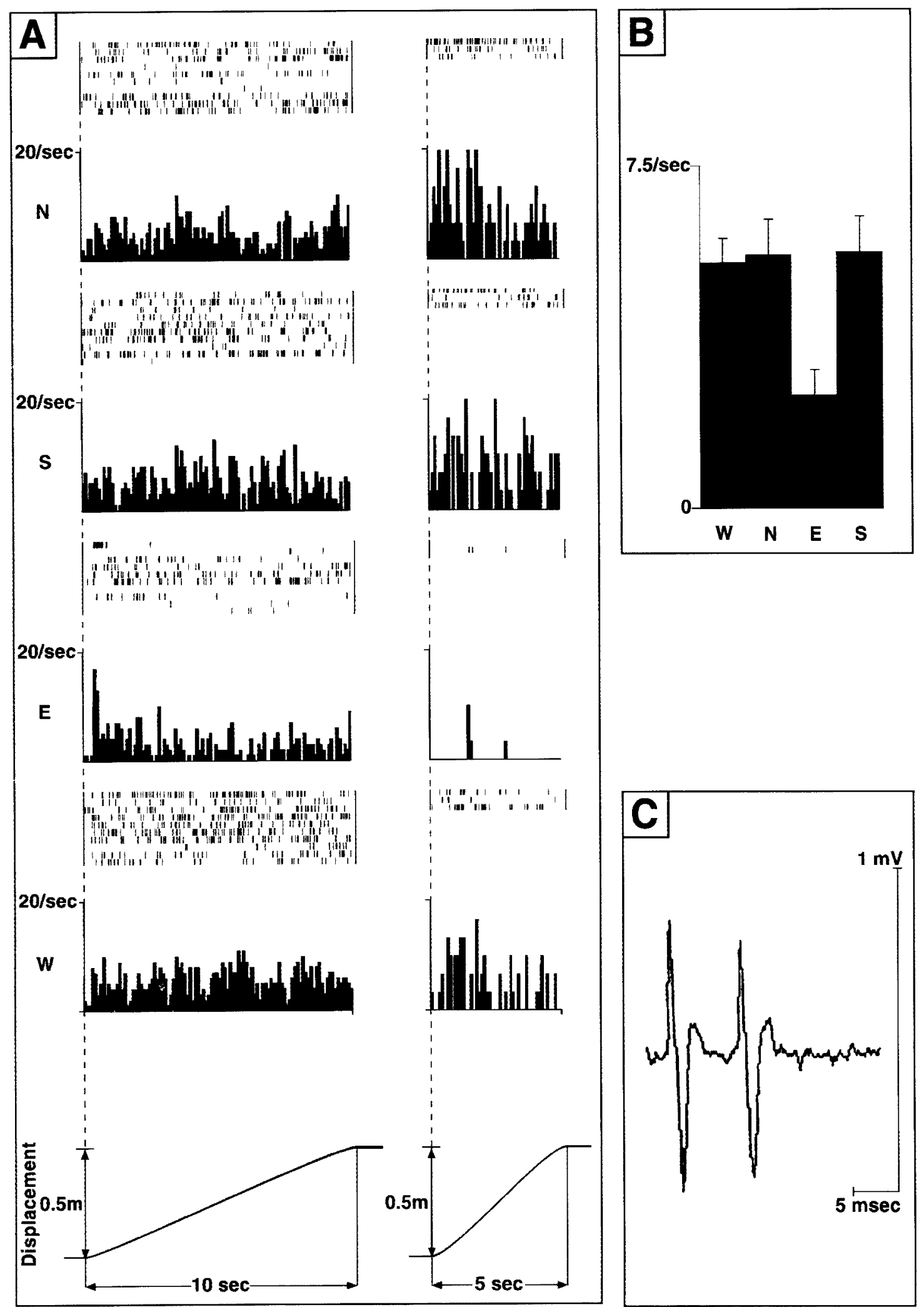

FIGURE 4. Location-selective decrease in average discharge rate during passive translations. A: Raster displays and histograms synchronized with translations along the respective walls of the experimental enclosure. Left and right columns show data from slow and fast movements, respectively. The timecourse of the movements is shown below. B: The overall average firing rate of this neuron during translations along the four respective walls. C: Typical filtered, digitized waveforms from this cell. (cell 3127) 

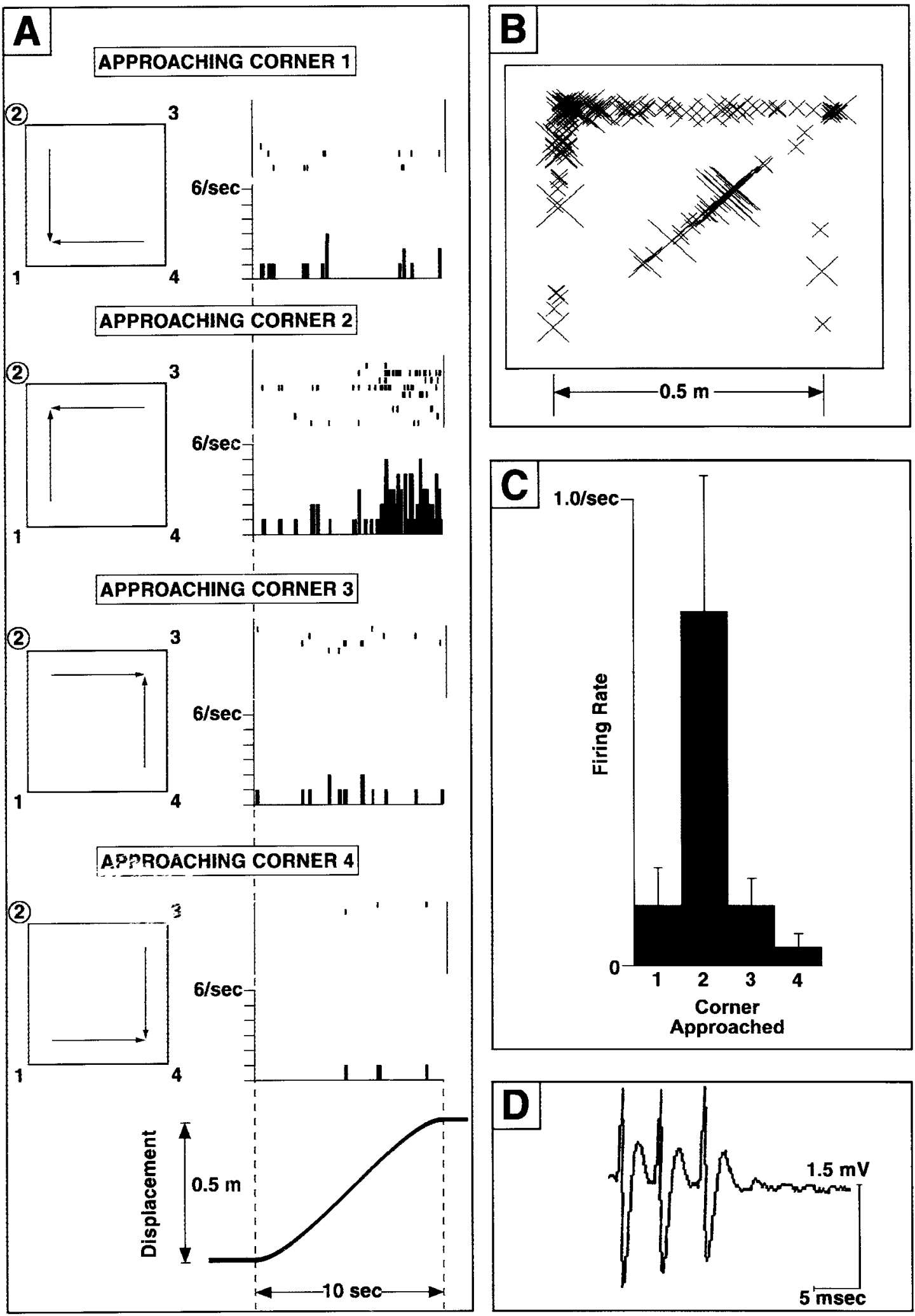

FIGURE 5. Another location-selective neuron recorded in the light. A: D ischarges of this neuron are synchronized with translations along the walls toward each of the respective corners. The trajectories included in the raster displays are schematized in the left column. The timecourse of the displacements is shown below. Firing occurred principally when the rat was in corner 2 during linear movement, rotations, and pauses. B: In this overhead view the discharges (Xs) principally occurred as the rat was transported along the west and north walls. C: 0 verall average firing rate during approaches along the walls to the four respective corners. D: Typical waveforms from this neuron. (cell 17127) 
population of neurons recorded for preferential discharges at any particular site, such as the corner where the reward was delivered.

\section{Movement-Selective Discharges}

In all six recording sessions in which analyses showed significant changes in discharge rate prior to, at the beginning, at the end, or after translations, the head of the rat was oriented toward the direction of movement, not in the other direction. In 5/6 of these sessions there was a significant reduction in firing rate in the period after the end of the movement (period 4) relative to at least one of the other periods. Two of these sessions included the same neuron recorded in the dark and the light, cell 27109. In this case the post hoc test results were identical for the two sessions: there was a significant reduction in firing rate during and after the movement.

Two neurons had a significant augmentation of firing rate during the onset of translation movements (cells 6055 and 16077)- one is shown in Figure 6. This hippocampal neuron was recorded in darkness. The firing rate increased significantly to 2.5 impulses/s during the first $2 \mathrm{~s}$ after initiation of linear translation compared with the final $2 \mathrm{~s}$ of movement and the $2 \mathrm{~s}$ pause afterwards. As the figure shows, several seconds after the start of movement the firing rate appears to decrease briefly, and then returns to background levels. Figure $6 \mathrm{~A}$ shows representative data from translations along only one of the walls. The histogram in Figure 6B summarizes data from this neuron for translations along all the walls over the entire session.

\section{Mixed Responses}

The majority of the neurons that had significant correlates in one analysis also had positive results in analyses of other types of movements (Table 1). The relationship between these results could not always be discerned. H owever, in other cases, this could be interpreted as a complex behavioral correlate. There was a wide variety in the combinations of correlates found, and no particular combination of independent correlates appeared more frequently than others. An example of a neuron with a complex correlate appears in Figure 7; the initial statistical analyses of this session (recorded under lit conditions) indicated that the discharge rate was significantly higher during rotations in corner 3 than in corner 1. H owever, this analysis pooled clockwise and counterclockwise rotations. In Figure 7A and B, the selectivity appears more pronounced for the counterclockwise rotations in corner 3 than for clockwise rotations. However, in both cases, the highest discharge rates correspond to when the rat was in corner 3 and the lowest were for corner 1 . Furthermore, Figure $7 \mathrm{C}$ confirms that the discharge rates were higher during the rotation than before the onset of rotation. Thus this neuron has a combination of location-, direction- and movement-selective correlates.

\section{Comparisons Between Recordings of the Same Neuron in Light and Dark Sessions}

Eleven cells were recorded in both light and darkness (18109, $2059,27109,30117,30118,5105,6055,7105,8107,8129$, and
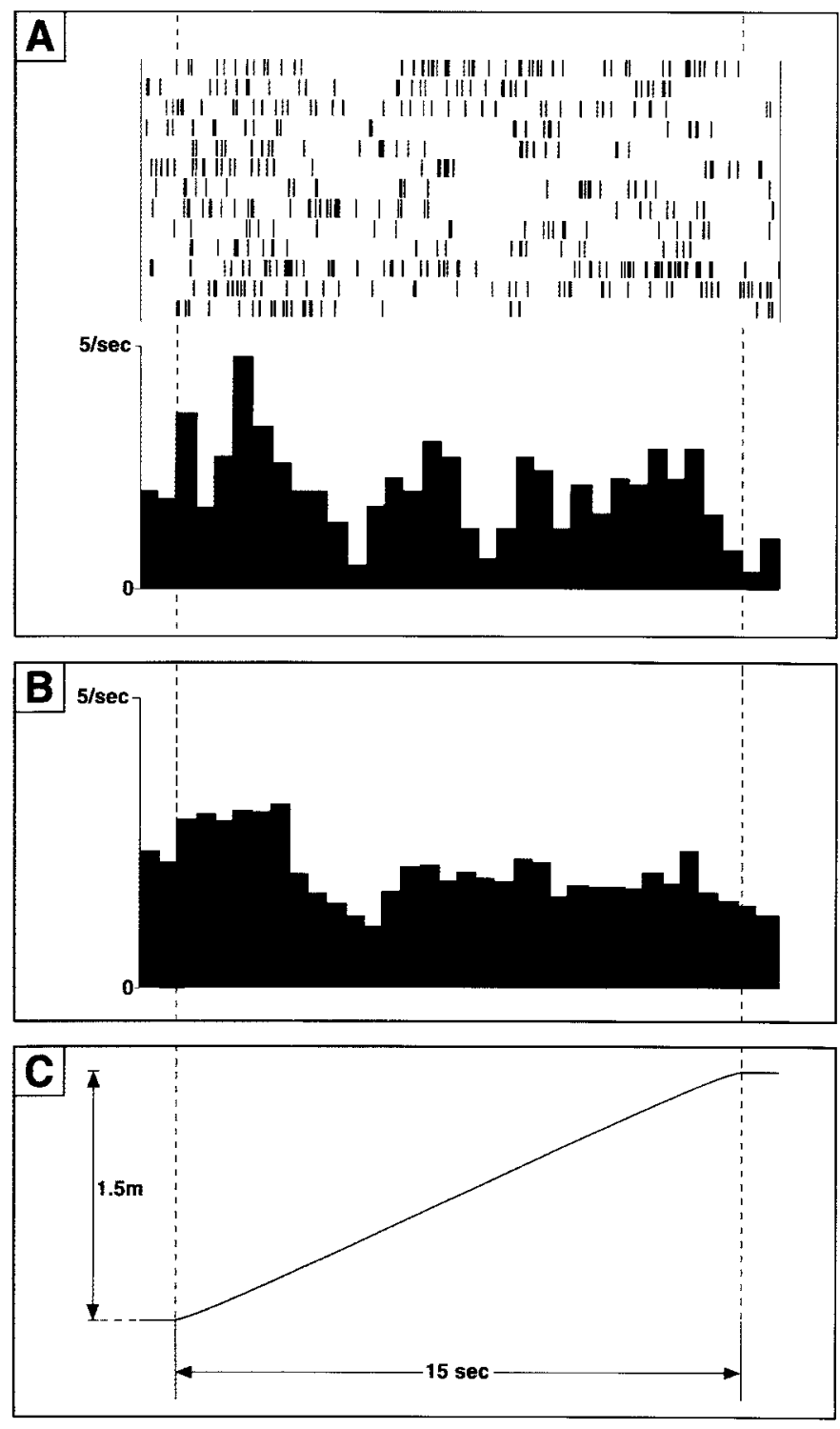

FIGURE 6. A neuron recorded in darkness that had significant increases in discharge rate at the beginning of linear displacements. A: D ata from translations along one of the walls. Rasters show spike times for individual trials and histograms summarize this in $0.5 \mathrm{~s}$ bins. B: Summary of discharges for this cell for all $\mathbf{5 2}$ translations along all four walls. C: Timecourse of the corresponding movements. (cell 16077)

9059), and two others were recorded successively in light, darkness, and then again in a light session (cells 2010 and 610). The firing properties of each of these neurons was compared between the light and dark sessions according to the number of common statistically significant results found in the eight analyses of Table 1. Remarkable similarities in AN OVA results from the same neuron recorded in light and dark sessions were found in four cells: 18109, 20108, 6105, and 8129. In neuron 18109 the AN OVA results (Table 1 ) were the same in the two sessions, and the post hoc test results were consistent, but not identical. In the other three cases at least $66 \%$ of the analyses showing significance were the same in light and dark sessions. 

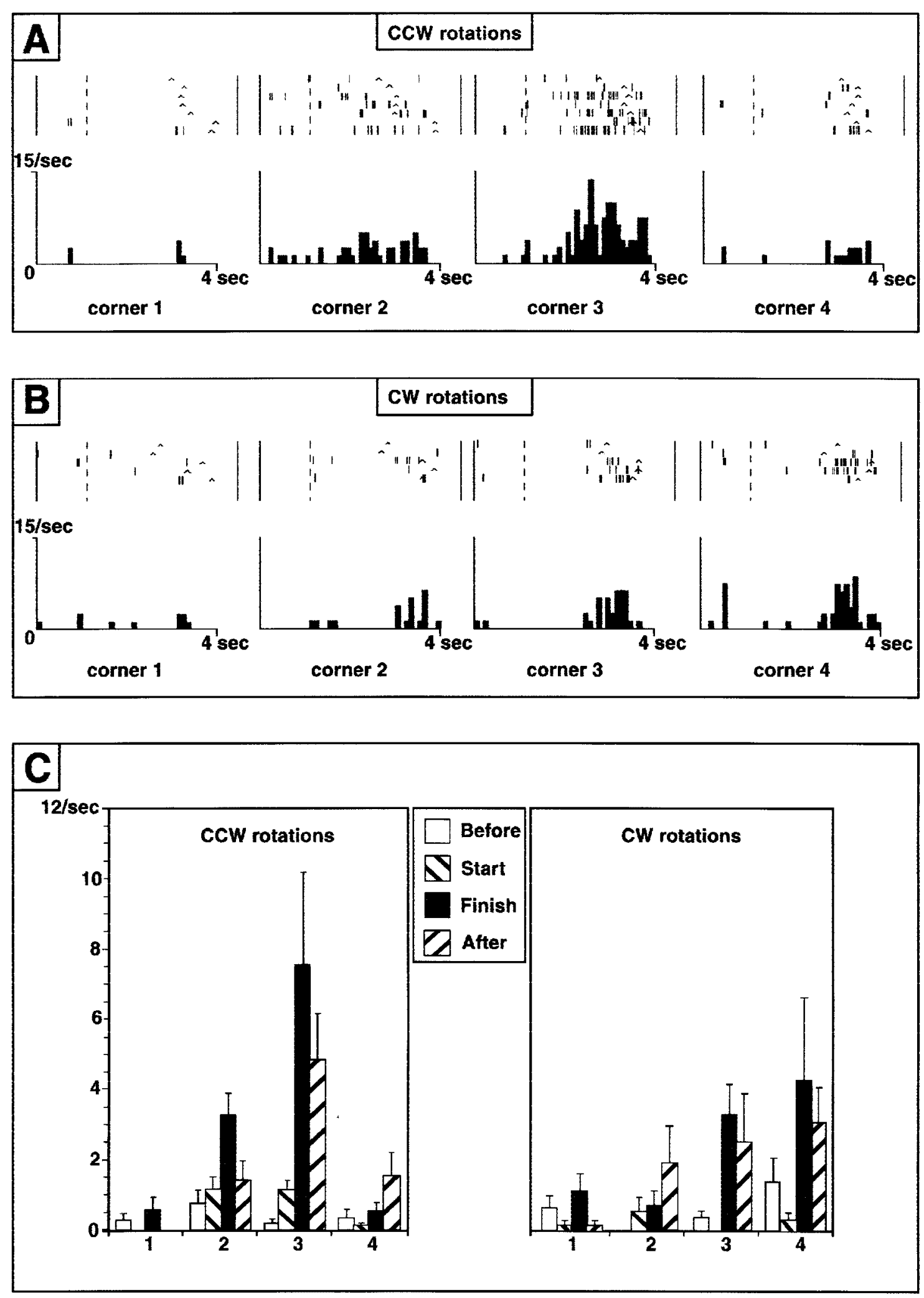

FIGURE 7. A hippocampal neuron that selectively discharged at the end of counterclockwise (CCW) rotations in corner 3. Raster displays are synchronized with the onset of movements (vertical dashed line). The inverted $V$ on each row indicates the instant that each rotation stopped. Rotations with several different starting and ending orientations are included. A: For the CCW rotations, the firing rate was higher in corner 3. B: T he firing rate increases tended to have the same spatial distribution but were much less pronounced for clockwise rotations. C: The data from A and B are shown as average firing rates for four periods of duration $1 \mathrm{~s}$ each: immediately prior to ("before") or after the onset ("start") of rotations, and immediately prior to ("finish") and after the end of the rotation. The greatest increases in firing rate occur prior to the end of the rotation. (cell 27109). 
A chi-square analysis was performed to compare the frequency of occurrence of the respective discharge correlates in the two experimental conditions- light and darkness. The proportion of cells with statistically significant responses were compared for the respective analyses (e.g., $34 \%$ of cells recorded in the light had significant differences for translations along the respective walls, while $31 \%$ of neurons recorded in darkness had the same result). The proportions were not significantly different for the two conditions $\left(\chi^{2}=1.19\right.$, $d f=7$, n.s. $)$.

\section{DISCUSSION}

The principal types of discharge correlates found in these hippocampal complex spike cells included selectivity for 1) position of the rat in the arena during translations, rotations, or pauses, 2) initiation or termination of movements independent of position, 3) heading direction, but only in limited region, and 4) direction of movements. Most neurons had more than one of these correlates.

\section{Inertial Stimulus-Related Activity in the Hippocampus}

The primary goal of this experiment was to test whether the hippocampus has access to sensory signals evoked by inertial stimuli. Although the semicircular canal and otolith organs respond selectively to rotational and linear acceleration, respectively, endorgans of nonvestibular sensory systems including visceral receptors, somatosensory proprioreceptors, and optic field flow detection could also be activated. M oreover, the latter two systems are known to send direct projections to the brainstem vestibular nuclei ( $H$ enn et al., 1974; Allum et al., 1976; Boyle and Pompeiano, 1980; Anastasopoulos and M ergner, 1982). Furthermore, movement information could be transmitted via kinesthesia as well as efferent collaterals of motor command signals. Such information could update representations of self-position (in the form of hippocampal "place cell" activity) as the animal moves about in darkness and could explain why hippocampal neurons can retain their location selectivity even after visual cues are no longer present (M cN aughton et al., 1989; Q uirk et al., 1990). This would enable a mouse dam to search for a pup along a complex multistage path but then return to the nest along a direct path in total darkness (M ittelstaedt and M ittelstaedt, 1980) - and would provide a physiological basis for the computations underlying "path integration." N eurons selective for the new position and orientation of the animal would thus be activated as the displacements of the animal are incrementally and vectorially added to the representation of the position previously occupied.

Two principal findings in the present study link path integration computations with hippocampal neuronal activity: 1) in the absence of visual and locomotor cues, hippocampal neurons can maintain location-selective and directionally modulated discharges, even after a complex and lengthy series of movements; and 2) hippocampal neurons also selectively discharge at the beginning or end of movements. The first argument is circumstantial: since visual, auditory, and olfactory cues were masked by the cloth, the only remaining cues (that we could ascertain) that could have updated these representations during the passive displacements were somatosensory and vestibular. In the intact animal, it is rather difficult to distinguish between the detection of inertial forces by these two sensory systems, although it seems likely that the vestibular system has better resolution under normal conditions. Although the location of the reward could also have served as a cue in some cases, selective discharges were also found for sides and corners of the arena opposite to the reward location. The discharges selective for the onset and end of movements could signal the dynamics of self-movements, which are essential for path integration computations. This would involve integrating velocity information over the timecourse of the movement. Although location-selective discharges are also found in entorhinal cortex (Q uirk et al., 1992), a principal source of projections to hippocampus, they are much more dependent on visual cues, and the firing fields are larger than those found in dorsal hippocampal CA3 and CA1. This suggests that important spatial information processing is also taking place within the hippocampus.

M ovement-selective discharges were also found in our studies of hippocampal dentateEEG in rats using the same protocol as in the current study (but with a different sequence of movements). Rhythmic slow activity in the high frequency range of the theta band $(6-9 \mathrm{~Hz})$ was induced at the onset of rotational as well as linear displacements in these restrained animals ( $G$ avrilov et al., 1995, 1996). Although the anatomical placement and the electrical characteristics of the electrodes in the present study were not optimal for recording theta EEG, evidence for this was also found here (Gavrilov, Wiener and Berthoz, unpublished observations). Theta EEG activity is also well known to occur in freely moving rats making active displacements. Thus the hippocampus has electrophysiological correlates of self-movements on both the single neuron and the ensemble level, and this could be involved in updating positional representations for navigation.

This is compatible with the results of M atthews et al. (1988), showing that an intact hippocampus is essential for passive rotational vestibular inputs to be taken into account for orientation in space. Rats were trained in a plus sign-shaped maze in which only one arm contained a reward. While confined to the center of the maze, the rats were rotated by a variable angle of up to 10 revolutions. Rats with vestibular lesions as well as those with hippocampal lesions had impaired performance relative to controls in finding the reward arm.

Although the location selectivity of the neurons recorded here was statistically significant, it extended over broader regions of the experimental room than the firing fields of dorsal hippocampal neurons reported in the freely moving rat (e.g., $O^{\prime} \mathrm{K}$ eefe and Conway, 1978). Since our protocol only permitted a limited variety of displacements in most places, it remains unknown whether this location selectivity would persist during other movements and with other heading angles. It is important to note that large firing fields have been found in neurons of the ventral hippocampus of freely moving rats (Jung et al., 1994). Thus the apparently larger firing field sizes found here are not in striking 
conflict with other results but are rather consistent with observations that this parameter varies even in unrestrained animals. Low-resolution location-selectivity has also been reported in hippocampal recordings of rats (O Iton et al., 1978; Thompson and Best, 1989), in areas receiving projections from area CA 1 such as entorhinal cortex (Q uirk et al., 1992), subiculum (Barnes et al., 1990; Sharp and Green, 1994), ventral striatum (Lavoie and M izumori, 1994), and anterodorsal striatum of the rat (Wiener, 1993). Ensemble coding can still yield highly accurate estimates of position from cells with poorly resolved spatial selectivity.

Foster et al. (1989) showed that location-selective discharges (firing fields) recorded in hippocampal neurons of freely moving rats become very weak when the rat is tightly restrained and returned to the sensitive location. However, normal activity returns when loosely restrained rats are again placed in this firing field. It seems that this result has been misinterpreted as providing evidence that in tightly restrained rats, hippocampal neurons have no firing fields. H owever, Foster et al. (1989) did not systematically record the cell activity while displacing the tightly restrained rats in all other parts of the arena; they tested only the (former) firing field and one other control location. The latter experiment thus leaves open the possibility that firing fields could have simply changed (in location, for example), rather than having vanished. The "very slight residual specificity" reported by Foster et al. (1989, p. 1581) in these conditions could correspond to some of the results found here. This issue remains unresolved for the moment since it was not the goal of the present experiments- and the cells recorded here were not tested in unrestrained rats.

In one group of neurons recorded in the present study, location-selectivity manifested through reductions in discharge rate (Fig. 4). This contrasts with the location-selective increases from the negligible background firing rate often reported for rat hippocampal complex spike cells. In hippocampal recordings of rats performing in a radial arm maze, O Iton et al. (1978) reported one category of neurons that showed reductions in firing rate as the rat visited one or several arms of the maze. O ' $\mathrm{M}$ ara et al. (1994) also reported that in the passively transported monkey, hippocampal neurons manifest behavioral correlates by reductions in ambient firing rate.

0 verall these results are remarkably similar to those obtained by 0 ' $M$ ara et al. (1994) in recordings in the macaque hippocampus. In these experiments, the animals were also restrained, but in a primate chair, which was mounted on a mobile robot of the same type as used here (but equipped with different software). The monkeys were then translated or rotated in a $2 \mathrm{~m} \times 2 \mathrm{~m} \times 2 \mathrm{~m}$ chamber in light and in darkness. In addition, the walls of the room could be displaced. M any of the response types shown here for rat hippocampal neurons were also found in the monkey study (albeit in different proportions), including selectivity for translations along only one wall of the arena, behavioral correlates maintained in light and dark conditions, discharges selective for rotation in one direction, and the initiation of linear translation. In an experimental paradigm different from that of 0 ' $M$ ara et al. (1994), O no et al. (1993) have also shown sel ective hippocampal unit discharges in seated monkeys as they were moved to certain positions of the experimental room.
In the O 'M ara et al. (1994) study as well as the present one, individual neurons had several different correlates, in some cases bearing no apparent relation to one another. For example, the post hoc tests (not shown) indicated that neuron 5105 discharged at a lower rate when the rat was transported along the west wall but also had a significantly lower discharge rate when the rat paused in the southeast corner. This recalls the multiple discharge correlates reported by $\mathrm{H}$ ampson et al. (1993) in rats performing a nonmatch to sample task requiring a bar press response. Almost 90\% of the hippocampal complex spike cells of the latter study fired selectively during the sample, the match response, and the reinforcement phase of the task. Among these, unique configurations of positional preferences (left versus right lever) were found for the different phases. Similar examples of multiple correlates can be found in the literature of hippocampal recordings from monkeys performing operant tasks. Perhaps the hippocampal neurons are linking these diverse events together with a logic that has not yet been fathomed.

The present report is the first to show that hippocampal discharge correlates are similar in monkeys and rats performing comparable tasks. This may be simply because the two species have never been tested in such similar paradigms, or because this protocol evokes certain characteristic modes of information processing common to the hippocampus in both species.

\section{Acknowledgments}

Thanks to V. Korshunov for help in constructing the electrode driver, P. LeBoucher for electronics, A. Treffel for mechanical engineering and fabrication, S. Schmied for programming the statistical analyses, F. Lacloche and F. Maloumian for figure preparation, R. Shibata for help with synchronizing the movement data, M.-A. Thomas and S. D outremer for histology, S. Lemarchand for animal care, D r. S. Thornton for advice on head fixation in awake rats, and Dr. A.B. Mulder for comments on the text. Supported by Human Frontiers, EC ESPRIT M U COM 3149 and 6615, EC Human Capital and Mobility Program, CN RS Programme Cognisciences. D r. G avrilov was supported by a grant from the Fondation pour la Recherche $M$ édicale.

\section{REFERENCES}

Allum JH J, Graf W, Dichgans J, Schmidt CL. Visual-vestibular interaction in the vestibular nuclei of the goldfish. Exp Brain Res 1976;26: 463- 485 .

Anastosopoulos D, M ergner T. C anal-neck interaction in vestibular nuclear neurons of the cat. Exp Brain Res 1982;46:269-280.

Barnes CA, M cN aughton BL, M izumori SJY, Leonard BW, Lin L-H. Comparison of spatial and temporal characteristics of neuronal activity in sequential stages of hippocampal processing. In: Progress in brain research (Storm-M athisen J, Zimmer J, $O$ ttersen O P, eds), pp 287-300. Amsterdam: Elsevier. 1990. 
Boyle R, Pompeiano 0. Responses of vestibulospinal neurons to sinusoidal rotation of neck. J N europhysiol 1980;44:633-649.

Christian EP, D eadwyler SA. Behavioral functions and hippocampal cell types: evidence for two nonoverlapping populations in the rat. J N europhys 1986;55:331-348.

Foster TC, Castro CA, M CNaughton BL. Spatial selectivity of rat hippocampal neurons: dependence on preparedness for movement. Science 1989;244:1580-1582.

Fox SE, Ranck JB J r. Electrophysiological characteristics of hippocampal complex-spike cells and theta cells. Exp Brain Res 1981;41:399-410.

Gavrilov V, Wiener SI, Berthoz A. The properties of hippocampal neurons observed during passive displacement of rats on a mobile robot. Eur J N eurosci 1994;7:137 (Abstract).

Gavrilov V, W iener SI, Berthoz A. Enhanced hippocampal theta EEG during whole body rotations in awake restrained rats. N eurosci Lett 1995;197:239-241.

Gavrilov V, W iener SI, Berthoz A. Whole-body rotations enhance hippocampal theta rhythmic slow activity in awake rats passively transported on a mobile robot. Ann N Y Acad Sci 1996;781:385-398.

$\mathrm{H}$ ampson RE, Heyser CJ, Deadwyler SA. Hippocampal cell firing correlates of delayed-match-to-sample performance in the rat. Behav Neurosci 1993;107:715-739.

H enn V, Young LR, Finley C. Vestibular nucleus units in alert monkeys are also influenced by moving visual fields. Brain Res 1974;74:144149.

Jung $\mathrm{M}, \mathrm{W}$ iener $\mathrm{SI}, \mathrm{McN}$ aughton $\mathrm{BL}$. Comparisons of spatial firing characteristics of units in dorsal and ventral hippocampus of the rat. J Neurosci 1994;14:7347-7356.

Korshunov VA. M iniature microdrive for extracellular recording of neuronal activity in freely moving animals. J Neurosci Methods 1995;57:77-80.

Lavoie, AM, M izumori, SJY. Spatial, movement- and reward-sensitive discharge by medial ventral striatum neurons of rats. Brain Res 1994; 638:157-168.

$\mathrm{M} \mathrm{CN}$ aughton BL, Leonard B, Chen LL. Cortical-hippocampal interactions and cognitive mapping: $\mathrm{A}$ hypothesis based on reintegration of the parietal and inferotemporal pathways for visual processing. Psychobiology 1989;17:230-235.

$M$ atthews BL, Campbell KA, Deadwyler SA. Rotational stimulation disrupts spatial learning in fornix-lesioned rats. Behav Neurosci 1988;102:35-42.

Mittelstaedt M, Mittelstaedt $\mathrm{H}$. Homing by path integration in a mammal. N aturwiss 1980;67:566-567.

Muller R. A quarter of a century of place cells. N euron 1996;17:813822.
0 'Keefe J. A review of the hippocampal place cells. Prog Neurobiol 1979;13:419-439.

0 'Keefe J, C onway DH. H ippocampal place units in the freely moving rat: why they fire where they fire. Exp Brain Res 1978;31:573-590.

0 'M ara SM, Rolls ET, Berthoz A, Kesner RP. N eurons responding to whole-body motion in the primate hippocampus. J N eurosci 1994;14: 6511-6523.

OIton DS, Branch M, Best PJ. Spatial correlates of hippocampal unit activity. Exp N eurol 1978;58:387-409.

Ono T, Nakamura K, Nishijo H, Eifuku S. Monkey hippocampal neurons related to spatial and non-spatial functions. J N europhys 1993;70:1516-1529.

Paxinos G, Watson C. The rat brain in stereotaxic coordinates, 2nd ed. Sydney: Academic Press 1986.

Q uirk GJ, M uller RU, Kubie JL. The firing of hippocampal place cells in the dark depends on the rat's recent experience. J N eurosci 1990;10: 2008-2017.

Quirk GJ, Muller RU, Kubie JL, Ranck JB Jr. The positional firing properties of medial entorhinal neurons: description and comparison with hippocampal place cells. J N eurosci 1992;12:1945-1963.

Sharp PE, Green C. Spatial correlates of firing patterns of single cells in the subiculum of the freely moving rat. J Neurosci 1994;14:23392356.

Sharp PE, Blair H T, Etkin D, Tzanetos D B. Influences of vestibular and visual motion information on the spatial firing patterns of hippocampal place cells. J N eurosci 1995;15:173-189.

Taube JS, M uller RU, Ranck JB Jr. H ead-direction cells recorded from the postsubiculum in freely moving rats. I. D escription and quantitative analysis. J N eurosci 1990;10:420-435.

Thompson LT, Best PJ. Place cells and silent cells in the hippocampus of freely behaving rats. J N eurosci 1989;9:2382-2390.

Wiener SI. Spatial and behavioral correlates of striatal neurons in rats performing a self-initiated navigation task. J N eurosci 1993;13:38023817.

Wiener SI. Spatial, behavioral and sensory correlates of hippocampal CA1 complex spike cell activity: implications for information processing functions. Prog N eurobiol 1996;49:335-361.

Wiener SI, Berthoz A. Forebrain structures mediating the vestibular contribution during navigation. In: Multisensory control of movement (Berthoz A, ed), pp 427-456. 0 xford: 0 xford University Press 1993.

W iener SI, Korshunov VA, G arcia R, Berthoz A. Inertial, substratal and landmark cue control of hippocampal CA1 place cell activity. Eur J N eurosci 1995;7:2206-2219. 